ISSN: 2386-3919 - e-ISSN: 2386-3927

DOI: https://doi.org/10.14201/et20173521736

\title{
DISEÑO Y CONFIABILIDAD DE UNA RÚBRICA PARA EVALUAR INFOGRAFÍAS DIDÁCTICAS
}

\section{Design and reliability of a didactic inphographic rubric assessment}

\author{
Yunuen Ixchel GuZMÁN-CEDILLO* \\ Diana Natalia LIMA-VILLEDA*** \\ José Manuel MEZA-CANO**** \\ Facultad de Psicología. Universidad Nacional Autónoma de México (México) \\ Correo-e: *yunuenixchel@hotmail.com \\ **diana.lima@iztacala.unam.mx \\ **Facultad de Estudios Superiores Iztacala, Universidad Nacional Autónoma de \\ México (México) \\ Correo-e: manuel.meza@iztacala.unam.mx
}

Recibido: 15-8-2016; Aceptado: 13-3-2017; Publicado: 30-11-2017

Ref. Bibl. YUNUEN IXCHEL GUZMÁN-CEDILLO, DIANA NATALIA LIMA-VILLEDA Y JOSÉ MANUEL MEZA-CANO. Diseño y confiabilidad de una rúbrica para evaluar infografías didácticas. Enseñanza \& Teaching, 35, 2-2017, 17-36.

\begin{abstract}
RESUMEN: El objetivo de esta investigación es describir el diseño, validación y medición de la confiabilidad de una rúbrica para evaluar la calidad de infografías didácticas. Los participantes fueron quince jueces relacionados con la elaboración de infografías didácticas. El diseño de la rúbrica se llevó a cabo en tres etapas: diseño, ajustes y determinación de la confiabilidad. La validez de contenido fue medida a través del porcentaje de acuerdo entre tres jueces por cada rubro. Así mismo se obtuvo un alpha de Krippendorff $(\alpha=.710)$ al evaluar previamente cinco infografías para evitar posibles contradicciones debidas a la redacción del rubro e indicador de desempeño. La consistencia interna fue determinada a través del coeficiente alpha de Cronbach $(\alpha=806)$ al evaluar 22 infografías didácticas. A los datos de seis evaluadores se aplicó un coeficiente de correlación intraclase ICC $(\alpha=.909)$ y un coeficiente
\end{abstract}


de Krippendorff ( $\alpha=$.538), ambos para medidas ordinales. La rúbrica se compone de nueve elementos, tres niveles de desempeño, definiciones por cada rubro, así como instrucciones de uso. De acuerdo a los resultados, la rúbrica tiene validez de contenido y es confiable para discriminar la calidad de infografías didácticas.

Palabras clave: infografía; rúbrica; evaluación; infografía didáctica.

SUMMARY: The objective of this study is to describe design, validity process and reliability of a rubric assessment to evaluate didactic infographics quality. Participants were fifteen judges who participate in different moments of elaboration rubric process; it was made in three process phases: design, settings and reliability determination. Content validity was obtained by percentage agreement between 3 judges by component of the rubric; likewise a Krippendorff's alpha were applied $(\alpha=.710)$ in pilot assessment with 5 infographics in order to set possible writings contradictions between components and criteria of performance. The intern consistence was determined by Cronbach's alpha $(\alpha=.806)$ in 22 infographics gradation. An Intraclass correlation coefficient ICC $(\alpha=.909)$ was applied to 6 judges qualifications also a Krippendorff's alpha $(\alpha=.538)$ both of them in ordinal levels. The rubric is composed by 9 components, 3 performance levels, definitions of each component and assignments how to use the rubric. Results suggest the rubric is valid and reliable to grade quality of didactic infographic.

Key words: inphographic; rubric; evaluation; teaching with inphographics.

\section{LAS RÚBRICAS DE EVALUACiÓN}

Una forma de evaluar productos de aprendizaje complejos es a través del uso de rúbricas. La rúbrica, en breve, es una matriz que se compone de criterios graduados cuya función es evaluar de manera integral todo el producto o desempeño a la vez que puede dar medidas particulares de los elementos o rubros que lo componen (Guzmán-Cedillo, Flores y Tirado, 2012; Tardif, 2006; Burton, 2015).

El National Institute for Learning Outcomes Assessment en Estados Unidos de América (Kuh, Jankowski, Ikenberry y Kinzie, 2014) reportó que los instrumentos de evaluación más utilizados en las aulas de las universidades de ese país son, en primer lugar, los exámenes (85\%), seguidos de las rúbricas (69\%). En general, en la práctica y la literatura educativa, se reporta un esfuerzo conjunto de desarrollo académico en la elaboración de rúbricas (un ejemplo es el proyecto VALUE en USA).

Cuando una rúbrica es validada y se cuida su confiabilidad, facilita la coherencia y la congruencia entre los evaluadores (Jonsson y Svingby, 2007). En ese sentido, para lograrlo es necesario cuidar el rigor en la elaboración de las rúbricas utilizadas en la evaluación de productos de aprendizaje (Reddy y Malini, 2010). 
Así mismo, un diseño mal llevado de los instrumentos de evaluación o poco entrenamiento del uso de la rúbrica son razones de un bajo nivel de confiabilidad (Panadero, 2013; Reddy y Malinni, 2010). En consecuencia, una inferencia es que los evaluadores deben ser lo suficientemente capacitados para lograr niveles aceptables de acuerdo (típicamente 70\% o superior) (Reddy y Malini, 2010). También se conocen experiencias positivas en la mejora de los aprendizajes, debido a que los criterios de evaluación son explícitos entre los actores del proceso educativo (Pufpaff, Clarke y Jones, 2015).

\section{PROBlema EN LA RIGUROSIDAD EN EL DISEÑO DE LAS RÚBRICAS}

Pese a todos los beneficios e incremento de uso de rúbricas, así como las herramientas que permiten su implementación en el aula como programas y aplicaciones informáticas (ejemplos: rubistar/forallrubrics), los estudios reportan una falta de transparencia en los procesos de elaboración en la parte de la validación y la medición de la confiabilidad. Eso hace pensar que el uso de la rúbrica de evaluación educativa se ha popularizado, pero falta una base científica de su elaboración.

Las rúbricas son más utilizadas porque logran una visión del desarrollo de los aprendizajes que busca promover la institución, estos instrumentos se acogen por los miembros de las facultades para dar dirección al desarrollo académico. Sin embargo, se ha detectado una falta de consistencia en el diseño y aplicación de estos instrumentos, esto refleja que el uso de las rúbricas no se ha profesionalizado.

En una estimación del problema de la falta de transparencia en las medidas psicométricas que reportan las rúbricas, Reddy y Melini (2010) y otros autores (Angell, 2015; Valverde, 2014) mencionan que si bien en los artículos se reporta la validez de las rúbricas utilizadas, se hace referencia a la claridad y el lenguaje apropiado, pero no es frecuente que se aborde la alineación entre los criterios comprendidos en la rúbrica y lo que se evalúa (validez de contenido); siendo poco evaluadas las facetas de la construcción de un instrumento (validez de constructo), así como la adecuación de las generalizaciones a otras actividades relacionadas (validez de criterio).

Peteers, Schmude y Steinmiller (2014), por su parte, atribuyen esta falta de transparencia en la validez y confiabilidad de las rúbricas al esfuerzo y tiempo que requieren.

El problema de transparencia de las propiedades psicométricas revelado en los estudios o experiencias reportadas revela que son muchos los profesores en diferentes campos que utilizan rúbricas sin considerar confiabilidad y sólo se preocupan por que sean comprensibles para ellos, tal vez otros profesores o acaso sus estudiantes (Razaei y Lovorn, 2010; Reddy y Melini, 2010).

Al respecto, Nicholson (2013) señala que en el caso de enfermería para evaluar juicios clínicos se ha dado un aumento de la validez de facie y menos atención a la confiabilidad. 
Reddy y Andrade (2010) ya señalaban en su estudio que el potencial benéfico en educación de las rúbricas ha sido demostrado, pero que se carece de métodos rigurosos en su construcción, sobre todo, en la atención a la validez y confiabilidad en diversos contextos educativos. Lo cierto es que las propiedades psicométricas de las rúbricas no han sido lo suficientemente especificadas por sus autores (Nicholson, 2013).

Al respecto, un hecho interesante es que a seis años del trabajo de Reddy y Andrade (2010) todavía son pocos los trabajos publicados concernientes a las propiedades psicométricas de las rúbricas que se presentan como instrumentos de evaluación (Doolen, 2015; Nicholson, 2013; Angell, 2015; Guzmán-Cedillo, Flores y Tirado, 2013; Valverde, 2014; Peeters et al., 2014; Houh, 2013; Panadero, 2013). De tal manera que puede pensarse que el uso de una rúbrica es intrascendente, ya que lograr una confiabilidad alta no es fácil, porque se debe tener cuidado de no sacrificar la validez para lograr mayores índices de confiabilidad (Razaei y Lovorn, 2010).

\section{MÉTODOS DE VALIDEZ Y CONFIABILIDAD PARA EL DISEÑO DE RÚBRICAS}

El proceso de validación de una rúbrica se ha realizado con mayor frecuencia a través de la validez de contenido, entendida como el juicio que realizan expertos en el tema al determinar si la muestra del comportamiento que se busca examinar es realmente representativa del dominio que va a ser evaluado (Angell, 2015). En la rúbrica, los jueces determinan si los rubros, indicadores o niveles de desempeño son pertinentes para evaluar los productos o los procesos elegidos como objeto de la evaluación. Por ello, la experiencia de los jueces es fundamental tanto en el conocimiento como en la evaluación del producto o desempeño. La calificación por parte de los jueces a los elementos de la rúbrica se realiza desde una escala de 0 y $1(0=$ no pertinente y 1 = es pertinente) o con el uso de una escala Likert de 4 puntos (en la que 1 es irrelevante y 4 es muy relevante y concreto) (Doolen, 2015; Angell, 2015; Nicholson, 2013; D’Antonni, 2009; Guzmán-Cedillo et al., 2013; Lee y Cherner, 2015).

Con respecto a la confiabilidad de la rúbrica y sus niveles de desempeño, se pueden calcular a través de las medidas estimadas (el consenso, la consistencia y la medida). El consenso se basa en la posibilidad de que los evaluadores lleguen al acuerdo sobre los niveles de variabilidad con respecto a lo analizado en la medición de una rúbrica. La consistencia asume que no es necesario que los evaluadores compartan un significado común de las posiciones o niveles de desempeño de la escala, sino qué tanto el evaluador es consistente consigo mismo al clasificar el fenómeno de acuerdo con su propia definición de la escala. Finalmente, la medida está basada en que se debe utilizar toda la información dada por los evaluadores (incluidas las posiciones en las que hay desacuerdo) para lograr un resumen de medida por cada evaluador (Guzmán Cedillo et al., 2012).

La razón principal de por qué validar y cuidar la confiabilidad de la rúbrica radica en que los criterios y expectativas se hacen explícitos para quienes deben emitir un juicio de calidad sobre el objeto a evaluar. En el caso de este documento, 
el objeto de evaluación refiere a la calidad de las infografías realizadas por los estudiantes.

\section{INFOGRAFÍAS}

La infografía es un elemento del periodismo digital, la cual es una respuesta ante la necesidad de captar lectores en pantalla y en línea (Valero, 2008). Habitualmente, comunica los temas a través de multimedia, llámense dibujos, fotografías o videos, los cuales forman parte de los signos convencionales abstractos y sonidos más o menos conocidos por los lectores.

Diferentes expertos en el tema, la definen como una representación gráfica de una explicación clara y sencilla (Valero, 2008), un tipo de comunicación informativa, un sistema funcional, además de la presentación tanto de análisis como de síntesis de la información o un despliegue visual de esta (Krauss, 2012; Minervini, 2005; Ortega et al., 2012).

\section{INFOGRAFÍAS DIDÁCTICAS}

Existe evidencia de que la infografía puede fungir como recurso de aprendizaje, ya que se tienen hallazgos que afirman que mejora la percepción de los estudiantes sobre la didáctica o bien de la forma en que facilita la adquisición de conocimientos por parte de las personas (Cabrera, 2013; García, 2014; Brigas, Gonçalves y Milheiro, 2013; Guzmán-Cedillo, Lima-Villeda y Ferreira-Rosa, 2015; Kibar y Akkoyunlu, 2014; Minervini, 2005; Muñoz, 2014; Reinhardt, 2007, 2010; Roney, Menjívar y Morales, 2015; Rueda, 2015; Vallejo, 2013).

El uso de la infografía como recurso educativo es reciente. En los estudios hay dos líneas identificables: 1. La infografía es un recurso educativo atractivo y 2. La realización por parte de los educandos (Martix y Hodson, 2014; Mendenhall y Summers, 2015; Roney et al., 2015; Rueda, 2015). En esta segunda vertiente se remarca la elaboración como oportunidad de promover la investigación, el análisis y la síntesis de la información, además de las competencias digitales, pues la elaboración de una infografía por parte de los estudiantes requiere la conformación de un objeto gráfico atractivo visualmente, además de descriptivo y claro. Así mismo, este organizador gráfico manifiesta un entendimiento del tema estudiado además de la posibilidad de su intercambio a través de las redes.

Las infografías de las organizaciones involucran un equipo de profesionales de distintas disciplinas (Centeno y Cabrera, 2005). A diferencia de ella «... la infografía didáctica es reflejo de un aprendizaje basado en el desarrollo de un proyecto educativo relacionado a los contenidos abordados en el proceso de formación que desemboca en la elaboración de la infografía realizada por estudiantes como producto de su actividad como aprendices...» (Guzmán-Cedillo et al., 2015: 965). 


\section{RÚBRICAS PARA EVALUAR INFOGRAFÍAS DIDÁCTICAS}

En relación con utilizar la rúbrica para evaluar infografías didácticas en diferentes niveles o formaciones educativas, se observa un número reducido de propuestas que la exponen como parte de la secuencia didáctica (Matrix y Hodson, 2014; Nuhoglu, 2014) o al menos proponen el uso de una lista de cotejo para brindar, compartir y clarificar estándares de calidad en el curso (Davidson, 2014). Son menos aún los trabajos que exponen las características psicométricas de estos instrumentos (Guzmán-Cedillo et al., 2015). Así mismo, en esta investigación se ubicaron 7 rúbricas abocadas a la evaluación de infografías didácticas expuestas como parte de una experiencia educativa mostrada en blogs, sitios o ponencias de congresos todas ellas en lengua inglesa, no así artículos de investigación educativa que den información sobre el conocimiento de las repercusiones en los aprendizajes al elaborar infografías didácticas o la sistematización en la construcción de una rúbrica para evaluarlas (Shroock, 2012).

Al respecto, tanto por la revisión de la literatura de infografías como las infografías realizadas por organismos internacionales, se sabe que de forma general reúnen ciertos elementos e indicadores que deben de ser tomados en cuenta para determinar su calidad y logro del objetivo a informar (Nuhoglu, 2014; Niebaum, Cunningham-Sabo, Carroll y Bellows, 2015; Valero, 2001 en González-Panacanowwski y Medina, 2009).

Las investigaciones subrayan elementos clave que determinan la calidad de la infografía, tales como: formato, diagramación, calidad de las imágenes, así como la información y la forma en que se expresa el conocimiento en la infografía.

Con respecto al formato, las características de presentación de una infografía se refieren a la representación de un conjunto gráfico, donde los textos se encuentran interrelacionados de forma precisa, y se promueve una armonía coherente entre el texto y la imagen, apreciando así una proporción entre datos e imágenes.

La diagramación está acorde con la lectura visual, que es simple y clara, cuya tipografía es cuidada en términos de diseño, forma, tamaño y las relaciones visuales que se establecen entre letras, números y símbolos.

La calidad de las imágenes utilizadas da el aspecto estético, las cuales mantienen la concordancia a nivel lingüístico y temporal, al utilizar imágenes que son el elemento central de toda infografía.

Los fines inherentes a la infografía en términos de contenido son considerados al ser claras, comprensibles, informativas y sintéticas. La expresión de ideas complejas en un formato visual digerible es el propósito de la infografía, por eso resulta necesario tratar de dar la información esencial con un mínimo de tiempo de lectura invertido y espacio al combinar las imágenes con palabras para incrementar así la comprensión y retención de la información, por ello cuentan una historia a manera de respuesta a las preguntas de qué, cuándo, cómo y por qué, respuestas soportadas todas ellas en información fidedigna y de los más recientes hallazgos en investigación científica (Niebaum y cols., 2015). 
A partir de estos antecedentes en el presente estudio se tuvo como planteamiento cómo diseñar una rúbrica válida y confiable para evaluar infografías didácticas. Por ello el objetivo del estudio es desarrollar una rúbrica de evaluación de infografías didácticas.

\section{MÉTODO}

En el caso de la presente investigación, se trata de construir una rúbrica que evalúe un producto dentro de una escala ordinal. Para ello, se retoma el método de elaboración propuesto por Gürsul y Keser (2009), adaptado por Guzmán-Cedillo et al. (2012), señalado como el proceso de elaboración (calibración) de rúbricas más utilizado (Yuan y Recker, 2015); porque es un proceso flexible, que se inicia con la definición de las características psicoeducativas a evaluar, los modelos de medición propuestos en la literatura científica y en su caso práctica (Enfermería, Música, Programación, etc.).

Todos esos antecedentes se utilizan como base para elaborar los rubros que los jueces expertos validan en términos de pertinencia (validez de contenido). Después, piden que los jueces utilicen la rúbrica para ubicar el producto de aprendizaje de los estudiantes en un nivel de desempeño para cada rubro a través de criterios o indicadores del desarrollo del aprendizaje o dominio, datos que sirven para calcular la consistencia interna del instrumento y la confiabilidad entre observadores obteniendo, así, una medición de la confiabilidad de la rúbrica en general o por rubro. Con la finalidad de ajustar la rúbrica las veces que se requiera.

\section{PARTICIPANTES}

Una muestra de máxima variación (Alaminos y Costa, 2006), compuesta por 15 jueces que utilizaron la rúbrica en las diferentes fases de diseño de la rúbrica. Nueve de ellos estudiantes de la licenciatura en Psicología de diferentes semestres, quienes participaron como voluntarios en el ajuste de los rubros y evaluación de infografías. Cinco jueces expertos en diferentes disciplinas (diseño, edición, sociología, psicología y tecnologías) que conocen y trabajan con la infografía didáctica.

Unidad de análisis: 46 infografías didácticas que evaluarían los jueces en diferentes momentos del ajuste de la rúbrica, 22 de ellas fueron las revisadas por los últimos 6 jueces estudiantes, quienes recibieron capacitación para el uso de la última versión de la rúbrica.

\section{PROCEDIMIENTO}

Constó de tres fases consecutivas: diseño, ajustes y determinación de confiabilidad. 


\subsection{Diseño}

En la fase de diseño se realizó la investigación documental y entrevistas, donde se buscaron ejemplos de infografías realizadas por organismos internacionales (Organización Panamericana de la Salud, Organización Mundial de la Salud, asociaciones y Secretarías de Salud). Se entrevistó a dos diseñadores gráficos sobre la evaluación de infografías y de contenidos digitales.

\subsection{Ajustes}

Desde la primera hasta la última versión (22) se realizaron varios ajustes con respecto a los comentarios de los diferentes jueces, de tal forma que de tener una primera versión con cuatro rubros y tres niveles de desempeño (avanzado, intermedio y en desarrollo) (Guzmán et al., 2015), después de las revisiones de 15 jueces, los análisis y comparaciones, se presenta en este artículo una rúbrica con instrucciones de uso, compuesta por nueve rubros todos ellos definidos, además de tres niveles de desempeño: principiante, avanzado y experto, así como la descripción de los criterios de desempeño por cada rubro y nivel que conforman los indicadores en la matriz (véase el anexo 1).

\subsection{Determinación de confiabilidad}

La rúbrica se utilizó por dos jueces docentes para evaluar previamente cinco infografías y ajustar posibles contradicciones debidas a la redacción del rubro e indicador de desempeños por medio del programa QDAminer versión 4.1 se calculó la confiabilidad de medida interjuez (alpha de Krippendorfff).

Realizados los ajustes se capacitó a seis jueces estudiantes para evaluar de forma independiente infografías didácticas; con la evaluación de cada juez por infografía se construyó la base de datos, a la cual se le aplicó un alpha de Cronbach a través del programa SPSS versión 15 y fue determinada la consistencia interna, así mismo a los datos de los seis observadores se les aplicó un coeficiente de correlación intraclase ICC y un coeficiente de alpha de Krippendorff, ambos para medidas ordinales (Hayes y Krippendorff, 2007; Prieto, Lamarca y Casado, 1998).

\section{Resultados}

En primera instancia se muestran los resultados de los acuerdos en la pertinencia de los rubros; después se dan los resultados del alpha de Krippendorff calculada con las medidas de dos jueces docentes al utilizar la rúbrica de evaluación por cada uno de los rubros y nivel de desempeño al evaluar 5 infografías, para cerrar con los análisis de confiabilidad de la rúbrica al ser utilizada por 6 jueces estudiantes evaluando 22 infografías. 


\subsection{Pertinencia de los rubros en el diseño de la rúbrica}

En la Tabla 1 se muestra el porcentaje de acuerdo sobre la pertinencia de los rubros calificados con 0 si no era pertinente y 1 si se le consideraba pertinente, calificados por tres jueces.

TABLA 1

Porcentaje de acuerdo sobre la pertinencia de los rubros para evaluar la infografía

\begin{tabular}{|l|c|}
\hline \multicolumn{1}{|c|}{ RUBRO } & PORCENTAJE DE ACUERDO \\
\hline Título & $100 \%$ \\
\hline Formato de texto & $100 \%$ \\
\hline Colores & $66 \%$ \\
\hline Integración & $66 \%$ \\
\hline Información & $100 \%$ \\
\hline Referentes & $100 \%$ \\
\hline Derechos de autor & $100 \%$ \\
\hline Ortografía y redacción & $100 \%$ \\
\hline Creatividad & $100 \%$ \\
\hline
\end{tabular}

\subsection{Ajustes a la rúbrica y consistencia de medida entre jueces}

Después de realizar modificaciones a la definición de colores e integración, se llevó a cabo una evaluación piloto de cinco infografías de forma independiente por dos jueces docentes, con un $70 \%$ de acuerdo total entre ellos (véase Tabla 2).

TABLA 2

Porcentaje de acuerdo entre jueces docentes en la evaluación piloto

\begin{tabular}{|c|c|c|c|}
\hline ACUERDO AUSENTE & ACUERDO PRESENTE & DESACUERDO & $\begin{array}{c}\text { PORCENTAJE } \\
\text { DE ACUERDO }\end{array}$ \\
\hline 32 & 17 & 20 & $70 \%$ \\
\hline
\end{tabular}

Estos resultados se discutieron entre los jueces y se afinaron los criterios de desempeño de los rubros por nivel (principiante, avanzado y experto); realizadas estas modificaciones se procedió a evaluar nuevamente las infografías de manera independiente, además de calcular el alpha de Krippendorff. Los resultados pueden observarse en la Tabla 3. 
TABLA 3

Acuerdo entre jueces docentes en la evaluación piloto

\begin{tabular}{|c|c|c|c|c|c|}
\hline & $\begin{array}{c}\text { ACUERDO } \\
\text { AUSENTE }\end{array}$ & $\begin{array}{c}\text { ACUERDO } \\
\text { PRESENTE }\end{array}$ & DESACUERDO & $\begin{array}{c}\text { PORCENTAJE } \\
\text { DE ACUERDO }\end{array}$ & $\begin{array}{c}\text { ALPHA DE } \\
\text { KRIPPENDORF }\end{array}$ \\
\hline Total & 71 & 36 & 18 & $85.60 \%$ & 0.712 \\
\hline
\end{tabular}

\subsection{Análisis de confiabilidad de la rúbrica}

Ya discutidos y afinados los indicadores con resultados menores a $80 \%$ de acuerdo (2, derechos de autor, así como ortografía y redacción, en nivel principiante), se capacitó a 6 estudiantes en el uso de la rúbrica, quienes llevaron a cabo la evaluación de 22 infografías didácticas.

A los datos obtenidos se aplicó un análisis estadístico de la confiabilidad de la escala a través de un alpha de Cronbach $(\alpha=.806$.) y un coeficiente de correlación intraclase por cada rubro. Los resultados se muestran en la Tabla 4.

TABLA 4

Estadísticos por rubro

\begin{tabular}{|l|c|c|c|}
\hline & $\begin{array}{c}\text { MEDIA DE LA ESCALA } \\
\text { SI SE ELIMINA } \\
\text { EL ELEMENTO }\end{array}$ & $\begin{array}{c}\text { ALFA DE CRONBACH } \\
\text { SI SE ELIMINA } \\
\text { EL ELEMENTO }\end{array}$ & $\begin{array}{c}\text { COEFICIENTE DE } \\
\text { CORRELACIÓN } \\
\text { INTRACLASE }\end{array}$ \\
\hline Título & 17.3664 & .796 & .420 \\
\hline Formato de texto & 17.6107 & .782 & .816 \\
\hline Colores & 17.6947 & .778 & .839 \\
\hline Integración & 17.5496 & .778 & .826 \\
\hline Información & 17.5649 & .763 & .801 \\
\hline Referentes & 18.0382 & .816 & .943 \\
\hline Derechos de autor & 18.5725 & .791 & .919 \\
\hline Ortografía y redacción & 17.8931 & .809 & .773 \\
\hline Creatividad & 17.8321 & .766 & .649 \\
\hline
\end{tabular}

Los resultados totales del ICC se muestran en la Tabla 5 y en la Tabla 6 los resultados de correlación interjuez. 
YUNUEN IXCHEL GUZMÁN-CEDILLO

DISEÑO Y CONFIABILIDAD DE UNA RÚBRICA PARA EVALUAR INFOGRAFÍAS DIDÁCTICAS

TABLA 5

Resumen de los elementos

\begin{tabular}{|l|c|c|c|c|c|c|c|}
\hline & $\begin{array}{c}\text { CORRELACIÓN } \\
\text { INTRACLASE (A) }\end{array}$ & \multicolumn{2}{|c|}{$\begin{array}{c}\text { INTERVALO DE } \\
\text { CONFIANZA 95\% }\end{array}$} & \multicolumn{4}{|c|}{ PRUEBA F CON VALOR VERDADERO 0 } \\
\cline { 2 - 8 } & $\begin{array}{c}\text { LÍMITE } \\
\text { INFERIOR }\end{array}$ & $\begin{array}{c}\text { LÍMITE } \\
\text { SUPERIOR }\end{array}$ & VALOR & GL1 & GL2 & SIG. & $\begin{array}{c}\text { LÍMITE } \\
\text { INFERIOR }\end{array}$ \\
\hline $\begin{array}{l}\text { Medidas } \\
\text { individuales }\end{array}$ & $.623(\mathrm{~b})$ & .524 & .721 & 10.936 & 62 & 310 & .000 \\
\hline $\begin{array}{l}\text { Medidas } \\
\text { promedio }\end{array}$ & $.909(\mathrm{c})$ & .869 & .940 & 10.936 & 62 & 310 & .000 \\
\hline
\end{tabular}

TABLA 6

Resultados de correlación interjuez

\begin{tabular}{|c|c|c|c|c|c|c|}
\hline & $\mathrm{J} 1$ & $\mathrm{~J} 2$ & $\mathrm{~J} 3$ & $\mathrm{~J} 4$ & $\mathrm{~J} 5$ & $\mathrm{J6}$ \\
\hline $\mathrm{j} 1$ & 1.000 & .708 & .746 & .595 & .475 & .762 \\
\hline $\mathrm{j} 2$ & .708 & 1.000 & .771 & .396 & .488 & .693 \\
\hline $\mathrm{j} 3$ & .746 & .771 & 1.000 & .578 & .587 & .756 \\
\hline $\mathrm{j} 4$ & .595 & .396 & .578 & 1.000 & .524 & .645 \\
\hline $\mathrm{j} 5$ & .475 & .488 & .587 & .524 & 1.000 & .627 \\
\hline $\mathrm{j} 6$ & .762 & .693 & .756 & .645 & .627 & 1.000 \\
\hline
\end{tabular}

Además, para determinar la confiabilidad de medida entre jueces se aplicó un coeficiente de alpha de Krippendorff ( $\alpha=.538$, véase Tabla 7), ambos para medidas ordinales.

TABLA 7

Resumen de la confiabilidad estimada por el coeficiente alpha de Krippendorff

\begin{tabular}{|c|c|c|c|c|c|c|}
\hline & ALPHA & LL95\%CI & UL95\%CI & UNIDADES & OBSERVADORES & PARES \\
\hline Ordinal & .5382 & .4425 & .6319 & 81 & 6 & 1044 \\
\hline
\end{tabular}

\section{DISCUSIÓN Y CONCLUSIONES}

Este trabajo abona a la línea de elaboración de rúbricas que se distinguen por tener un proceso de desarrollo riguroso y sistemático, que se suma a los esfuerzos en ciencias educativas por proporcionar instrumentos de evaluación útiles que 
ayuden a docentes y estudiantes a mejorar sus procesos de enseñanza aprendizaje. La rúbrica desarrollada sin duda puede ser útil para docentes que ya utilizan la infografía didáctica.

Al igual que en el desarrollo de exámenes o escalas válidas y consistentes, se requiere de un metódico diseño y uso de las rúbricas, así como su combinación con otras formas de evaluación que den evidencia del logro de objetivos de aprendizaje y análisis comparativo que demuestren de manera clara su efecto, tanto en las prácticas educativas como en los aprendizajes de los estudiantes (Arcario, Eynon, Klages y Polnariev, 2013; Bartolomé, Martínez-Figueira y Tellado, 2014; De la Serna y Bergman, 2014; Etkina et al., 2006; Jonsson y Svingby, 2007; Kuh et al., 2014). Además, sirve de retroalimentación para el docente en términos de la didáctica y el desarrollo de los aprendizajes de los estudiantes.

Así mismo, en la revisión de literatura científica se han ubicado propuestas del uso de la rúbrica para evaluar tareas complejas como: escritos colaborativos, ensayos individuales, reportes de investigación, toma de decisiones profesionales al resolver un caso, debates sobre dilemas o situaciones desestructuradas. Pese a ello, en la revisión documental realizada no se encontró una rúbrica que pudiese ayudar a los autores de este trabajo a evaluar infografías didácticas.

Si bien la infografía didáctica es una herramienta educativa cada vez más utilizada, en la revisión documental se ubicaron solo siete rúbricas de evaluación dentro de secuencias didácticas alojadas en la Red con criterios de desempeño que podían guiar a los estudiantes en su realización, pero ningún artículo que transparentase el proceso de construcción de las rúbricas para evaluar las infografías.

Para el objetivo de este trabajo (describir el diseño, validación y medición de la confiabilidad de una rúbrica de evaluación de infografías que permita evaluar la calidad de las infografías didácticas), los globales tanto del alpha de Cronbach (.806) de la rúbrica, como el Icc promedio (.909) de los jueces fueron buenos.

Sin embargo, el alpha de Krippendorff reportada $(\alpha=.538)$ con las mediciones de los estudiantes aún se encuentra en "nivel de construcción del conocimientom (De Weve, Van Keer, Schellens y Valcke, 2007; Hayes y Krippendorff, 2007), lo que sugiere considerar que este estudio pertenece a un campo educativo nuevo, por ello es importante afinar el proceso de capacitación y medir nuevamente este coeficiente de confiabilidad entre observadores para que los criterios o descriptores de evaluación que la integran sean lo más claros posibles para los estudiantes.

En ese sentido, se logra desarrollar una primera versión de una rúbrica consistente de evaluación de infografías didácticas, la cual, a la luz de los datos, debe afinarse, pero es la primera propuesta con etapas de diseño y sistematización de la rúbrica para evaluar la calidad de las infografías, lo cual es en sí mismo un aporte para la docencia que busca tener elementos y criterios para evaluar una de las actividades ya frecuentes en el medio educativo.

Con el objeto de que arroje datos coherentes entre evaluadores, tal como lo menciona Burton en su reciente artículo de 2015, así como Soiferman (2015) y 
Jonsson y Svingby (2007), quienes señalan que una rúbrica con validez y confiabilidad promueve congruencia entre los actores del proceso educativo.

El diseño de una rúbrica es un proceso complejo donde a veces los rubros no brindan indicadores comprensibles para realizar el producto o desempeño de aprendizaje, donde se asignan puntuaciones discretas por cada rubro o se necesita un criterio más integral para poder comprender el objeto en su contexto y el desarrollo que lleva a cabo el estudiante para complementar con los niveles de desempeño todas esas categorías que componen la rúbrica. Además de la temporalidad o momentos que se dan en el curso en donde la rúbrica es una herramienta de evaluación formativa para promover el desarrollo del dominio que se busca como meta de aprendizaje.

Al respecto, Guzmán-Cedillo et al. (2012) mencionan que tener una rúbrica donde se cumplan criterios de validez y confiabilidad apunta a hacer explícitos los criterios de evaluación para quienes deben emitir un juicio respecto del objeto evaluado. Entonces los estudiantes tienen presentes los criterios de evaluación en todo momento de tal forma que puedan valorar el proceso de construcción de su producto final.

Así mismo, en este contexto, se sugiere que, en posteriores investigaciones, pueda analizarse la secuencia didáctica o el trabajo en equipo desarrollado por los estudiantes en relación al nivel de desempeño logrado por sus infografías. Además de considerar el campo de elaboración de infografías en salud un área fértil de trabajo en investigación educativa, así como en las áreas involucradas: Medicina, Psicología, Trabajo social, entre otras.

\section{REFERENCIAS BIBLIOGRÁFICAS}

Alaminos, A. y Costa, J. (2006). Elaboración, análisis e interpretación de encuestas, cuestionarios de escalas de opinión. Marfil. Ice/Vicerrectorado de Calidad y Armonización Europea Universidad de Alicante. Recuperado de: https://rua.ua.es/dspace/ bitstream/10045/20331/1/Elaboraci\%C3\%B3n,\%20an\%C3\%A1lisis\%20e\%20interpretaci\%C3\%B3n.pdf.

Angell, K. (2015). The application of reliability and validity measures to assess the effectiveness of an undergraduate citation rubric. Behavioral \& Social Sciences Librarian, 34 (1), 2-15. doi: 10.1080/01639269.2015.996481.

Arcario, P.; Eynon, B.; Klages, M. y Polnariev, B. A. (2013). Closing the loop: how we better serve our students through a comprehensive assessment process. Metropolitan Universities Journal, 24 (2), 21-37. Recuperado de http://www.laguardia.edu/uploadedFiles/ Main_Site/Content/Divisions/AA/Assessment/Docs/Arcario\%20Eynon\%20Klages\%20 \%20Polnariev\%20-Closing\%20the\%20Loop\%20MUJ\%20-\%20fall\%202013.pdf.

Bartolomé, A.; Martínez-Figueira, E. y Tellado-González, F. (2014). La evaluación del aprendizaje en red mediante blogs y rúbricas: ¿complementos o suplementos? REDU. Rev. de Docencia Universitaria, 12 (1), 159-176. Recuperado http://red-u.net/redu/index.php/ REDU/article/view/767. 
Brigas, J.; Gonçalves, J. y Milheiro, S. (2013). Infographics in the education context. En C. Sousa, P. Tadeu y T. Paiva (Coords.). Proceedings Bookfor the Conference on Enabling Teachers for Entrepreneurship Education (pp. 51-56). Guarda: Polytechnic Institute. Recuperado de https://run.unl.pt/handle/10362/10687.

Burton, K. (2015). Continuing My Journey on Designing and Refining Criterion-Referenced Assessment Rubrics. Journal of Learning Design, 8 (3), 1-13. Recuperado de: http:// files.eric.ed.gov/fulltext/EJ1083802.pdf.

Cabrera, I. M. (noviembre 2013). Elaboración de infografías digitales como apoyo didáctico para el aprendizaje en la licenciatura en Psicología. EDUTEC. Recuperado de: http:// edutec2013.ac.cr/memoria/ponencias/cabrera_119.pdf.

Centeno, A. y Cabrera, M. (2005). La infografía multimedia para la web como recurso periodístico. Tesis: Licenciatura. Universidad Central, Caracas.

D'Antoni, A. V.; Zipp, G. P. y Olson, V. G. (2009). Interrater reliability of the mind map assessment rubric in a cohort of medical students. BMC Medical Education, 9 (1), 1.doi: 10.1186/1472-6920-9-19.

Davidson, R. (2014). Three Investigations in Which Students Present their Results in Infographics. The Science Teacher, 34-39. Recuperado de: http://crisp.southernct.edu/ images/8/82/Infographics_NSTA.pdf.

De la Serna, M. y Bergman, M. E. (2014). Evaluación formativa con e-rúbrica: aproximación al estado del arte. REDU. Revista de Docencia Universitaria, 12 (1), 15-22. Recuperado de http://red-u.net/redu/index.php/REDU/article/view/807.

De Wever, B.; Van Keer, H.; Schellens, T. y Valcke, M. (2007). Applying multilevel modelling to content analysis data: Methodological issues in the study of role assignment in asynchronous discussion groups. Learning and Instruction, 17 (4), 436-447. Recuperado de: https://biblio.ugent.be/publication/386211/file/6798582.pdf.

Doolen, J. (2015). Psychometric Properties of the Simulation Thinking Rubric to Measure Higher Order Thinking in Undergraduate Nursing Students. Clinical Simulation in Nursing, 11 (1), 35-43. doihttp://dx.doi.org/10.1016/j.ecns.2014.10.007.

Etkina, E.; Van Heuvelen, A.; White-Brahmia, S.; Brookes, D. T.; Gentile, M.; Murthy, S. y Warren, A. (2006). Scientific abilities and their assessment. Physical Review special topics-physics education research, 2 (2). Recuperado de: http://journals.aps.org/prstper/abstract/10.1103/PhysRevSTPER.2.020103.

González-Pacanowski, T. y Medina, P. (2009). Valor comunicacional de la infografía. El Profesional de la Información, 4 (18), 413-420. Recuperado de http://www.elprofesionaldelainformacion.com/contenidos/2009/julio/08.pdf.

Guzmán-Cedillo, Y.; Flores, R. C. y Tirado, F. (2012). La evaluación de la competencia argumentativa en foros de discusión en línea a través de rúbricas. Innovación Educativa, 60 (12), 17-40. Recuperado de https://dialnet.unirioja.es/servlet/articulo?codigo = 4219776.

Guzmán-Cedillo, Y.; Flores, R. y Tirado, F. (2013). Desarrollo de la competencia argumentativa en foros de discusión en línea: una propuesta constructivista. Anales de Psicología, 29, 907-916. Recuperado de http://scielo.isciii.es/scielo.php?pid = S0212-97282013000300030yscript $=$ sci_arttextytlng $=$ en .

Guzmán-Cedillo, Y. I.; Lima-Villeda, N. y Ferreira-Rosa, S. (2015). La experiencia de elaborar infografías didácticas sobre diversidad sexual. Revista Latina de Comunicación Social, 70, 961-981. Recuperado de http://www.revistalatinacs.org/070/paper/1080/50es.html. 
Gürsul, F. y Keser, H. (2009). The effects of online and face to face problem based learning environments in mathematics education on student's academic achievement. Procedia-Social and Behavioral Sciences, 1 (1), 2817-2824. doi:10.1016/j.sbspro.2009.01.501.

Hayes, A. F. y Krippendorff, K. (2007). Answering the call for a standard reliability measure for coding data. Communication Methods and Measures, 1, 77-89. doi: 10.1080/19312450709336664.

Jonsson, A. y Svingby, G. (2007). The use of scoring rubrics: Reliability, validity and educational consequences school of teacher education. Educational Research Review, 2 (2), 130-144. Recuperado de https://www.pdx.edu/education/sites/www.pdx.edu.education/files/Scoring_Rubrics_(Reliability,Validity,Consequences).pdf.

Kibar, P. y Akkoyunlu, B. (2014). A New Approach to Equip Students with Visual Literacy Skills: Use of Infographics in Education. En S. Kurbanoğlu, S. Špiranec, E. Grassian, D. Mizrachi y R. Catts (Eds.). Information Literacy. Lifelong Learning and Digital Citizenship in the 21st Century: Vol. 492 Communications in Computer and Information Science (pp. 456-465). doi: 10.1007/978-3-319-14136-7_48.

Krauss, J. (2012). More than words can say inphographics. Learning and leading with technology, 5 (39), 10-14. Recuperado de http://crossroadsnorthlibrary.pbworks.com/w/ file/fetch/53867217/Learning\%20and\%20Leading\%20-\%20February\%202012.pdf.

Kuh, G. D.; Jankowski, N.; Ikenberry, S. O. y Kinzie, J. (2014). Knowing what students know and can do: The current state of student learning outcomes assessment in us colleges and universities. Urbana, IL: University of Illinois and Indiana University, National Institute for Learning Outcomes Assessment (NILOA). Recuperado de: http://utsa.edu/ students/sanews/2014/issue05/files/2013SurveyReportFinal.pdf.

Lee, C.-Y. y Cherner, T. S. (2015). A comprehensive evaluation rubric for assessing instructional apps. Journal of Information Technology Education: Research, 14, 21-53. Recuperado de: http://www.jite.org/documents/Vol14/JITEV14ResearchP021-053Yuan0700. pdf.

Matrix, S. y Hodson, J. (2014). Teaching with inphographics: practicing new digital competencies and visual literacies. Journal of pedagogic development, 4 (2), 17-27.

Mendenhall, S. y Summers, S. (2015). Designing Research: Using Infographics to Teach Design Thinking. Composition.Journal of Global Literacies. Technologies and Emerging Pedagogies, 3 (1), 359-371. Recuperado de http://joglep.com/files/4514/3899/1848/10._ Article-Mendenhall_and_Summers-Designing_Research.pdf.

Minervini, M. A. (2005). La infografía como recurso didáctico. Revista Latina de Comunicación Social. Recuperado de: http://www.ull.es/publicaciones/latina/200506minervini. pdf.

Muñoz, G. (2014). Uso didáctico de las infografías. Espiral. Cuadernos del Profesorado, 7 (13), 37-43. Recuperado de: http://www.cepcuevasolula.es/espiral/articulos/ESPIRAL_VOL_7_N_14_ART_4.pdf.

Nicholson, P.; Griffin, P.; Gillis, S.; Wu, M. y Dunning, T. (2013). Measuring nursing competencies in the operating theatre: Instrument development and psychometric analysis using Item Response Theory. Nurse education today, 33 (9), 1088-1093. doi:10.1016/j. nedt.2012.04.008.

Niebaum, N.; Cunningham-Sabo, L.; Carroll, J. y Bellows, L. (2015). Infographics: An Innovative Tool to Capture Consumers Attention. Journal of Extension, 53 (6). Recuperado de http://www.joe.org/. 
Nuhoğlu, N. y Akkoyunlu, B. (2014). A New Approach to Equip Students with Visual Literacy Skills: Use of Infographics in Education. En Information Literacy. Lifelong Learning and Digital Citizenship in the 21st Century (pp. 456-465). Springer International Publishing.

Ortega, C.; Rincón, L. I.; Izquierdo, V.; Llanos, J.; Forero, Á.; Chacón, A.; Chavarro, J.; Rodríguez, G. R. y Carvajal, R. (2012). Ensayo e informe sobre la infografía. Bogotá: Corporacion Universitaria UNITEC. Recuperado de http://carlosmoreno.info/upn/2012/ ensayo665BD.pdf.

Panadero, E. y Romero, M. (2012). Uso de las rúbricas evaluación para fomentar el aprendizaje autorregulado/autónomo. Revista del Congrés Internacional de Docència Universitària i Innovació (CIDUI), 1 (1). Recuperado de: http://cidui.org/revista-cidui12/index. $\mathrm{php} / \mathrm{cidui} /$ article/view/458/448.

Peeters, M. J.; Schmude, K. A. y Steinmiller, C. L. (2014). Inter-rater reliability and false confidence in precision: Using standard error of measurement within PharmD admissions essay rubric development. Currents in Pharmacy Teaching and Learning, 6 (2), 298-303. Recuperado de: https://www.researchgate.net/profile/Michael_Peeters2/ publication/259524983_Inter-Rater_Reliability_and_False_Confidence_in_Precision_ using_standard_error_of_measurement_within_PharmD_admissions_essay_rubric_ development/links/00b49530b80494b679000000.pdf.

Prieto, L.; Lamarca, R. y Casado, A. (1998). La evaluación de la fiabilidad en las observaciones clínicas: el coeficiente de correlación intraclase. Medicina Clínica, 110 (4), 142-145. Recuperado de: http://www.elsevier.es/en-revista-medicina-clinica-2-articulo-la-evaluacion-fiabilidad-las-observaciones-2202.

Pufpaff, L. A.; Clarke, L. y Jones, R. E. (2015). The Effects of Rater Training on Inter-Rater Agreement. Mid-Western Educational Researcher, 27 (2), 117. Recuperado de: http:// www.mwera.org/MWER/volumes/v27/issue2/v27n2-Pufpaff-FEATURE-ARTICLE.pdf.

Reddy, M. y Melini, H. (2010). A review of rubric use in higher education. Assessment E Evaluation in Higher Education, 4 (35), 435-448. Recuperadode http://www.informaworld. $\mathrm{com} /$ openurl?genre $=$ articleyid $=$ doi:10.1080/02602930902862859.

Reinhardt, V. N. (2007). Infografía didáctica. Producción interdisciplinaria de infografías para la diversidad cultural. Tesis de doctorado. Universidad de Palermo. Recuperado de: http://www.palermo.edu/dyc/maestria_diseno/pdf/tesis.completas/10\%20Reinhardt.pdf.

Reinhardt, V. N. (2010). Infografía didáctica. Cuadernos del Centro de Estudios en Diseño y Comunicación [Ensayos], 11 (31), 119-191. Recuperado de: http://www.scielo.org.ar/ pdf/ccedce/n31/n31a03.pdf.

Rezaei, A. R. y Lovorn, M. (2010). Reliability and validity of rubrics for assessment through writing. Assessing writing, 15 (1), 18-39. doi:10.1016/j.asw.2010.01.003.

Roney, C.; Menjívar, E. y Morales, H. L. (2015). Elaboración de infografías: hacia el desarrollo de competencias del siglo xxI. Diálogos. Recuperado de: http://rd.udb.edu.sv:8080/ xmlui/bitstream/handle/123456789/1456/2\%20elaboraciondeinfografias.pdf?sequence $=1$.

Rueda, A. (2015). Uso de la infografía en los entornos virtuales personalizados para el proceso de enseñanza-aprendizaje sobre el álgebra booleana. Vivat Academia, 130, 37-47. Recuperado de: http://www.vivatacademia.net/index.php/vivat/article/view/617/141.

Schrock, K. (2012). Inphographic rubric. Recuperado de: http://www.schrockguide.net/ uploads/3/9/2/2/392267/schrock_infographic_rubric.pdf. 
Soiferman, L. K. (2015). Understanding the Effectiveness of Rubrics from the Students' Point of View. Online Submission. Recuperado de http://eric.ed.gov/?id = ED558553.

Tardif, J. (2006). L'évaluation des compétences: documenter le parcours de développement. Montréal: Chenelière-éducation.

Valero, J. L. (2008). La infografía digital en el ciberperiodismo. Revista Latina de Comunicación Social. Recuperado de: http://www.ull.es/publicaciones/latina/08/42_799_65_ Bellaterra/Jose_Luis_Valero.html. 492-504.

Vallejo, C. (2013). Infografias y competencia digital. Recuperado de: http://recursostic. educacion.es/observatorio/web/es/cajon-de-sastre/38-cajon-de-sastre/1091-infografias-y-competencia-digital?format $=$ pdf.

Valverde, J. (2014). El uso de e-rúbricas para la evaluación de competencias en estudiantes universitarios. Estudio sobre fiabilidad del instrumento. Revista de Docencia Universitaria, 12 (1), 49-79. Recuperado de: http://red-u.net/redu/index.php/REDU/article/ view/724.

Yuan, M. y Recker, M. (2015). Not All Rubrics Are Equal: A Review of Rubrics for Evaluating the Quality of Open Educational Resources. The International Review of Research in Open and Distributed Learning, 16 (5). Recuperado de: http://files.eric.ed.gov/fulltext/ EJ1077781.pdf. 


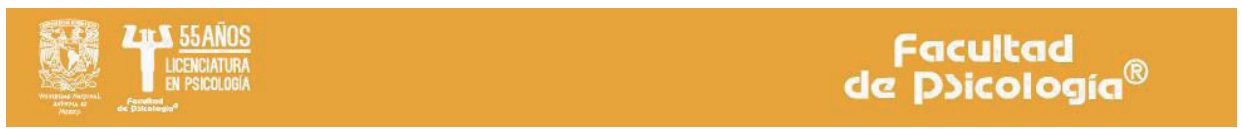

\section{Rúbrica para evaluar infografías didácticas}

Introducción. Dos de las competencias referidas por la UNESCO (2010) para los estudiantes del siglo XXI son la competencia informacional y la literacidad visual, una de las estrategias que promueven al mismo tiempo las dos competencias es la conformación de una infografía didáctica. Su elaboración se caracteriza por contener diferentes dimensiones para lograr dar un mensaje al lector.

En ese sentido se ha creado este instrumento con la finalidad de que puedas evaluar los alcances y mejoras de la infografía elaborada como resultado de una investigación documental con el objetivo de proyectarla de la forma más clara posible para la audiencia definida.

Objetivo de la rúbrica. Evaluar infografías didácticas realizadas por estudiantes después de llevar a cabo una investigación documental con el fin de mostrar sus hallazgos por medio de esta herramienta gráfica.

Instrucciones. Realiza una revisión general del instrumento, para después, con la infografía que vas a evaluar, respondas la primera sección e identifiques por cada dimensión o rubro en la rejilla un nivel de desempeño mostrado en su realización.

La primera columna contempla las dimensiones a evaluar en la infografía (título, formato, colores, integración, información, referentes, derechos de autor, ortografía y creatividad). Los niveles de desempeño repartidos en las tres columnas restantes buscan reflejar el descriptor de la infografía por cada dimensión ya sea experto, avanzado o novato.

\section{Primera sección}

Responde estas dos preguntas abiertas: ¿Cuál es el objetivo de la infografía?

¿Cuáles son los comentarios que tienes sobre la infografía? 


\section{Segunda sección}

Rúbrica para evaluar infografías didácticas

\begin{tabular}{|c|c|c|c|}
\hline \multirow{2}{*}{$\begin{array}{l}\text { DIMENSIÓN A } \\
\text { EVALUAR }\end{array}$} & \multicolumn{3}{|c|}{$\begin{array}{l}\text { NIVEL DE DESEMPEÑO MOSTRADO AL REALIZAR LA INFOGRAFÍA } \\
\text { POR CADA DIMENSIÓN }\end{array}$} \\
\hline & EXPERTO (3) & AVANZADO (2) & PRINCIPIANTE (1) \\
\hline $\begin{array}{l}\text { Título. } \\
\text { Nombre de la } \\
\text { infografía. }\end{array}$ & $\begin{array}{l}\text { El título atrae al lector, } \\
\text { es congruente y } \\
\text { abarca todas las ideas } \\
\text { de la información } \\
\text { tratada. }\end{array}$ & $\begin{array}{l}\text { El título abarca } \\
\text { algunas de las ideas } \\
\text { principales contenidas } \\
\text { en la infografía. }\end{array}$ & $\begin{array}{l}\text { El título es inexistente } \\
\text { o no tiene relación } \\
\text { con el contenido. }\end{array}$ \\
\hline $\begin{array}{l}\text { Formato de texto. } \\
\text { Tipo, forma y color de } \\
\text { letra utilizado. }\end{array}$ & $\begin{array}{l}\text { El tipo, color y tamaño } \\
\text { de letra utilizado } \\
\text { permite una lectura } \\
\text { fluida de la información. }\end{array}$ & $\begin{array}{l}\text { En segmentos el } \\
\text { tamaño, color o el tipo } \\
\text { de letra complican la } \\
\text { lectura de información. }\end{array}$ & $\begin{array}{l}\text { El tipo de letra es muy } \\
\text { variado o tan pequeño } \\
\text { que complica la lectura } \\
\text { ágil. }\end{array}$ \\
\hline $\begin{array}{l}\text { Colores. } \\
\text { Combinaciones } \\
\text { realizadas. }\end{array}$ & $\begin{array}{l}\text { La selección de todos } \\
\text { los colores y las texturas } \\
\text { utilizadas ha sido eficaz, } \\
\text { incrementando la } \\
\text { visibilidad del mensaje } \\
\text { de la infografía. }\end{array}$ & $\begin{array}{l}\text { La mitad de las } \\
\text { texturas y los colores } \\
\text { seleccionados ayudan } \\
\text { a la visibilidad del } \\
\text { mensaje de la infografía. }\end{array}$ & $\begin{array}{l}\text { Las texturas o los } \\
\text { colores seleccionados } \\
\text { dificultan la lectura del } \\
\text { mensaje de la infografía. }\end{array}$ \\
\hline $\begin{array}{l}\text { Integración. } \\
\text { Articulación de } \\
\text { recursos gráficos* y } \\
\text { textuales. } \\
\text { "Las imágenes, las } \\
\text { gráficas, las formas, } \\
\text { las figuras. }\end{array}$ & $\begin{array}{l}\text { Todos los } \\
\text { componentes ya sean } \\
\text { los gráficos y los textos } \\
\text { están relacionados de } \\
\text { tal forma que unifican } \\
\text { el mensaje de la } \\
\text { infografía. }\end{array}$ & $\begin{array}{l}\text { Los componentes se } \\
\text { relacionan, sin embargo, } \\
\text { hay un gráfico o un } \\
\text { texto utilizado que no } \\
\text { se integra o segmenta } \\
\text { el mensaje de la } \\
\text { infografía. }\end{array}$ & $\begin{array}{l}\text { Los gráficos o los textos } \\
\text { están desarticulados, } \\
\text { resulta difícil su } \\
\text { integración en el } \\
\text { mensaje de la infografía. }\end{array}$ \\
\hline $\begin{array}{l}\text { Información. } \\
\text { Contenido del } \\
\text { mensaje de la } \\
\text { infografía. }\end{array}$ & $\begin{array}{l}\text { El contenido se } \\
\text { encuentra organizado } \\
\text { en secciones al } \\
\text { utilizar subtítulos para } \\
\text { distinguirlas, dando una } \\
\text { secuencia de lectura. } \\
\text { El texto es suficiente, } \\
\text { los datos relevantes } \\
\text { y las imágenes se } \\
\text { relacionan con el tema } \\
\text { que se presenta. }\end{array}$ & $\begin{array}{l}\text { El contenido muestra } \\
\text { cierta organización, } \\
\text { aunque no se } \\
\text { identifican las secciones } \\
\text { de forma clara, o no } \\
\text { hay subtítulos para } \\
\text { distinguirlas. } \\
\text { El texto es insuficiente, } \\
\text { los datos son poco } \\
\text { relevantes o las } \\
\text { imágenes poco se } \\
\text { relacionan con el tema. }\end{array}$ & $\begin{array}{l}\text { El texto es cargado o } \\
\text { tiene información e } \\
\text { imágenes que poco } \\
\text { aportan a la claridad del } \\
\text { tema tratado. }\end{array}$ \\
\hline $\begin{array}{l}\text { Referentes. } \\
\text { Sustento de la } \\
\text { información. }\end{array}$ & $\begin{array}{l}\text { La información es } \\
\text { veraz y sustentada } \\
\text { en fuentes confiables } \\
\text { (expertos, artículos } \\
\text { u organizaciones) } \\
\text { y se encuentran } \\
\text { correctamente } \\
\text { referenciadas con un } \\
\text { estilo de citación. }\end{array}$ & $\begin{array}{l}\text { Realiza referencias } \\
\text { en fuentes confiables } \\
\text { de información } \\
\text { (expertos, artículos u } \\
\text { organizaciones) aunque } \\
\text { no se sigue ningún } \\
\text { estilo de citación. }\end{array}$ & $\begin{array}{l}\text { Se incluyen links de sus } \\
\text { referentes o no se citan } \\
\text { las fuentes utilizadas. }\end{array}$ \\
\hline
\end{tabular}




\begin{tabular}{|c|c|c|c|}
\hline \multirow{2}{*}{$\begin{array}{l}\text { DIMENSIÓN A } \\
\text { EVALUAR }\end{array}$} & \multicolumn{3}{|c|}{$\begin{array}{l}\text { NIVEL DE DESEMPEÑO MOSTRADO AL REALIZAR LA INFOGRAFÍA } \\
\text { POR CADA DIMENSIÓN }\end{array}$} \\
\hline & EXPERTO (3) & AVANZADO (2) & PRINCIPIANTE (1) \\
\hline $\begin{array}{l}\text { Derechos de autor. } \\
\text { Crédito a la autoría de } \\
\text { los elementos de la } \\
\text { infografía. }\end{array}$ & $\begin{array}{l}\text { Se identifica el nombre } \\
\text { del autor(a) de la } \\
\text { infografía y además se } \\
\text { da el reconocimiento } \\
\text { de la autoría de las } \\
\text { imágenes a través de } \\
\text { una nota o leyenda. }\end{array}$ & $\begin{array}{l}\text { Se identifica el nombre } \\
\text { o los nombres de los/as } \\
\text { autores/ o bien se da el } \\
\text { crédito correspondiente } \\
\text { a las imágenes } \\
\text { utilizadas. }\end{array}$ & $\begin{array}{l}\text { No se identifica el } \\
\text { nombre del autor/es } \\
\text { de la infografía ni se da } \\
\text { crédito a los autores de } \\
\text { las imágenes. }\end{array}$ \\
\hline $\begin{array}{l}\text { Ortografía y } \\
\text { redacción. } \\
\text { Acentuación, } \\
\text { gramática, coherencia } \\
\text { y cohesión textual. }\end{array}$ & $\begin{array}{l}\text { Su ortografía es } \\
\text { correcta, utiliza los } \\
\text { signos de puntuación } \\
\text { adecuadamente, la } \\
\text { redacción es clara y } \\
\text { ayuda a la comprensión } \\
\text { del mensaje. } \\
\end{array}$ & $\begin{array}{l}\text { La ortografía es correcta } \\
\text { y se hace un uso } \\
\text { adecuado de los signos } \\
\text { de puntuación, aunque } \\
\text { la redacción podría } \\
\text { mejorar la comprensión } \\
\text { del mensaje. }\end{array}$ & $\begin{array}{l}\text { Se encuentran algunos } \\
\text { errores ortográficos y } \\
\text { la redacción dificulta la } \\
\text { claridad del mensaje. }\end{array}$ \\
\hline $\begin{array}{l}\text { Creatividad. } \\
\text { Demostración de un } \\
\text { estilo propio. }\end{array}$ & $\begin{array}{l}\text { La infografía muestra la } \\
\text { idea personal del autor } \\
\text { cuando transforma la } \\
\text { información analizada } \\
\text { en una propuesta visual } \\
\text { al distribuir todos los } \\
\text { elementos en el espacio } \\
\text { de la infografía. }\end{array}$ & $\begin{array}{l}\text { En la infografía } \\
\text { hay segmentos } \\
\text { que muestran } \\
\text { poco tratamiento o } \\
\text { transformación de la } \\
\text { información analizada } \\
\text { y reflejada en su } \\
\text { propuesta visual. }\end{array}$ & $\begin{array}{l}\text { La infografía manifiesta } \\
\text { el uso de contenido } \\
\text { de otros, sin dar } \\
\text { tratamiento a la } \\
\text { información analizada } \\
\text { en su propuesta visual. }\end{array}$ \\
\hline
\end{tabular}

